\title{
ANK3 gene expression in bipolar disorder and schizophrenia
}

Katrine Verena Wirgenes, * Martin Tesli,* Elin Inderhaug, Lavinia Athanasiu, Ingrid Agartz, Ingrid Melle, Timothy Hughes, Ole Andreas Andreassen and Srdjan Djurovic

\section{Summary}

ANK3 gene variants have consistently been associated with bipolar spectrum disorder and schizophrenia spectrum disorder. However, the relevance of its encoded protein, ankyrin-3, in these disorders remains elusive. Here, we show that ANK3 gene expression in blood is significantly increased in bipolar disorder and schizophrenia compared with healthy controls. Additionally, we identified potential cis-acting expression quantitative trait loci located close to the transcription start site of one of the isoforms of the gene. These findings suggest that ANK3 mRNA is an interesting marker for further investigation of the underlying mechanisms in psychotic disorders.

\section{Declaration of interest}

None.
ANK3 is among the most frequently reported risk genes in bipolar disorder, ${ }^{1}$ with further evidence for association with schizophrenia, ${ }^{2}$ suggesting a common genetic mechanism in these two clinically related disorders. ANK3 sequence variants have been associated with clinical characteristics of bipolar disorder and schizophrenia, including impaired cognitive function ${ }^{3}$ and mood dysregulation. ${ }^{4}$ Gene variants are also associated with disturbed brain connectivity, ${ }^{5}$ and ankyrin-3 is expressed throughout the brain, ${ }^{6}$ with functions related to clustering of sodium and potassium channels, axodendritic polarity and action potential generation. Despite indications of altered gene expression, ${ }^{3,7}$ it is still unclear whether $A N K 3$ mRNA levels are altered in bipolar disorder and schizophrenia. Here we determined ANK3 mRNA levels in peripheral blood in individuals with bipolar disorder, with schizophrenia and in healthy controls. We also investigated potential cis-acting expression quantitative trait loci (eQTLs) in the ANK3 gene.

\section{Method}

Our sample (total $n=779$ ) comprised 227 patients with bipolar disorder (type $1 n=147$, type $2 n=65$ and bipolar disorder not otherwise specified $n=15$ ) (BD group), 273 patients with schizophrenia (schizophrenia $n=209$, schizophreniform disorder $n=19$ and schizoaffective disorder $n=45$ ) (SZ group) according to the Structured Clinical Interview for DSM-III-R (SCID), ${ }^{8}$ as well as 279 healthy controls. All participants were of White Northern European ethnicity. See online data supplement and Table DS1 for further details.

Quantitative reverse transcription polymerase chain reaction was performed using TaqMan Gene Expression Assays (ANK3: Hs00241738_m1; Life Technologies Corporation, Carlsbad, California, USA) and the gene GUSB (glucuronidase beta) as an endogenous control. Genotyping was performed in a subset of 685 individuals with Affymetrix Genome-Wide Human SNP array 6.0 (Affymetrix Inc., Santa Clara, California, USA) and additional imputation conducted with $\mathrm{MACH}$ using the European samples available in the Phase I release of the 1000 Genomes project (www.sph.umich.edu/csg/abecasis/MACH/download/1000G-PhaseIInterim.html). Further information on RNA measurement,

*These authors contributed equally to this work. genotyping procedures and imputation is presented in the online supplement.

Analysis of covariance (ANCOVA) was selected to investigate ANK3 mRNA level differences between participants with bipolar disorder, those with schizophrenia and healthy controls in the IBM SPSS software package for Windows, version 20. ANK3 mRNA was entered as a dependent variable and diagnostic spectrum (bipolar disorder, schizophrenia and healthy controls) as an independent variable, with age and gender as covariates. To further elucidate the differences in mRNA level between participants with bipolar disorder, those with schizophrenia and healthy controls, logistic regression was performed with mRNA, age and gender as predictors. Additionally, ANCOVA analyses were performed in males and females separately for main diagnostic spectra, as well as in the total sample for the diagnostic subcategories. Association analyses between 2398 imputed ANK3 single nucleotide polymorphisms (SNPs) and mRNA levels in a subset of 685 participants were performed with a linear regression model, with gender and age as covariates, using PLINK (version 1.07; http://pngu.mgh.harvard.edu/purcell/plink/). Additional information is presented in the online data supplement.

\section{Results}

The mRNA levels differed significantly between the BD, SZ and healthy control groups $\left(P=2.8 \times 10^{-4}\right.$; Fig. 1 and online Fig. DS1). The effect size (partial eta squared) was 0.021 for diagnostic spectrum (online Table DS2). The post hoc logistic regression tests showed significantly higher mRNA levels in the BD group $\left(P=9.0 \times 10^{-5}\right.$, odds ratio $\left.(\mathrm{OR})=2.29\right)$ and the $\mathrm{SZ}$ group $(P=0.010, \mathrm{OR}=1.73)$ compared with the healthy control group, but there was no significant difference between the BD and SZ group $(P=0.13, \mathrm{OR}=1.37)$. Results from the post hoc logistic regression analysis are presented in online Table DS3. The results remained largely the same when analysed in males and females separately (online Table DS4, Table DS5 and Fig. DS2). With respect to the diagnostic subcategories, there were significantly higher mRNA levels in bipolar type 1 disorder, bipolar type 2 disorder and schizophrenia compared with healthy controls, and significantly higher mRNA levels in bipolar type 2 disorder than in schizophrenia and schizophreniform disorder (online Table DS6, Table DS7 and Fig. DS1). ANK3 SNPs close to one of the 


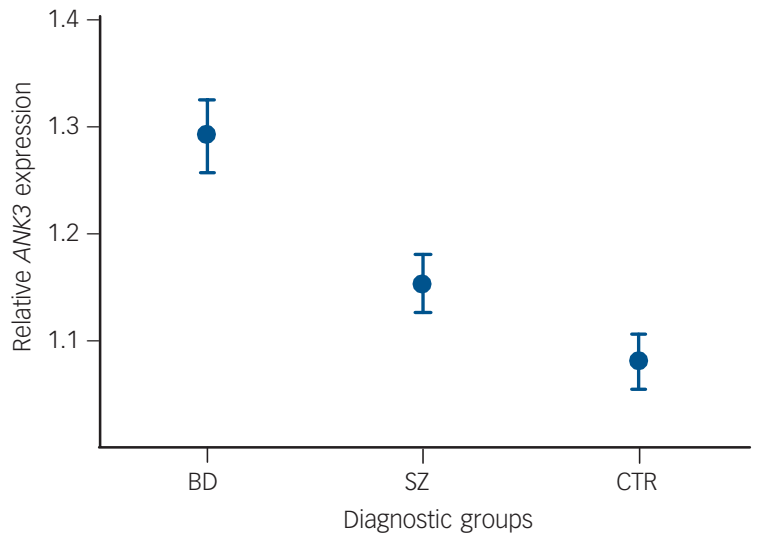

Fig. 1 ANK3 mRNA expression levels (mean (s.d. $=1$ ) value) according to diagnostic spectrum.

ANK3 mRNA levels are significantly higher in bipolar spectrum disorder (BD) $\left(P=9.0 \times 10^{-5}\right.$, odds ratio $\left.(O R)=2.29\right)$ and schizophrenia spectrum disorder (SZ) $\left(P=0.010, \mathrm{OR}^{\prime}=1.73\right)$ compared with healthy controls (CTR).

transcription start sites were significantly associated with ANK3 mRNA levels in the total subsample as well as in the BD, SZ and healthy control groups separately (online Table DS8 and Fig. DS3).

\section{Discussion}

We found ANK3 gene expression levels to be significantly increased in the $\mathrm{BD}$ and $\mathrm{SZ}$ groups compared with healthy controls. This effect was more pronounced for bipolar disorder than schizophrenia, with higher ANK3 expression in the affective end (bipolar type 2 disorder) than in the schizophrenia end of the psychosis spectrum (schizophrenia and schizophreniform disorder) (Table DS6, Table DS7 and Fig. DS1). Additionally, potential cis-acting eQTLs were identified. These results are in line with sequence variant findings, as ANK3 SNPs have been associated with both bipolar disorder and schizophrenia, albeit with stronger evidence in the former. Thus, increased ANK3 mRNA might be involved in a common pathophysiological mechanism underlying these disorders, supporting a continuum model of psychosis. This is in accordance with a finding of elevated ANK3 mRNA levels in peripheral blood in bipolar disorder, ${ }^{7}$ although post-mortem brain findings of down-regulation of ANK3 mRNA in superior temporal gyrus in individuals with schizophrenia $(n=46)$ has also been reported. ${ }^{3}$

Measurement of ANK3 mRNA in peripheral blood may not correlate with central nervous system (CNS) expression, but there is evidence for correlation between levels of transcripts expressed in blood and multiple CNS tissues. ${ }^{9}$

The effect sizes for SNP associations with psychiatric disorders are very low $(\mathrm{OR}<1.2)$, providing no clinical relevance. The current effect sizes for ANK3 mRNA level in bipolar disorder $(\mathrm{OR}=2.29)$ and schizophrenia $(\mathrm{OR}=1.73)$ were larger, indicating that mRNA level is a more robust marker than sequence variants for further assessment of underlying mechanisms in psychotic disorders. ANK3 mRNA level might be useful for investigations of the relevance of the ANK3 gene in brain networks, pharmacological interventions and clinical phenotypes in bipolar disorder and schizophrenia.

\section{Funding}

The work was supported by the Research Council of Norway, the South-East Norway Regional Health Authority and KG Jebsen Foundation.

\section{Acknowledgements}

We thank the patients and controls for their participation in the study, and the health professionals who facilitated our work. We also thank Thomas D. Bjella for assistance with professionals who facilitated our work. We also thank Thomas D. Bjella for assistance
the database and Lars J. Hansson for performing the RNA measurement procedures.

Katrine Verena Wirgenes, MD, Martin Tesli, MD, PhD, NORMENT, KG Jebsen Centre for Psychosis Research, Institute of Clinical Medicine, University of Oslo, and Division of Mental Health and Addiction, Oslo University Hospital, Oslo, Norway; Elin Inderhaug, BSC, Lavinia Athanasiu, PhD, NORMENT, KG Jebsen Centre for Psychosis Research, Institute of Clinical Medicine, University of Oslo, and Division of Mental Health \& Addiction and Department of Medical Genetics, Oslo University Hospital, Oslo, Norway; Ingrid Agartz, MD, PhD, NORMENT, KG Jebsen Centre for Psychosis Research, Institute of Clinical Medicine, University of Oslo; Division of Mental Health and Addiction, Oslo University Hospital; and Department of Psychiatric Research, Diakonhjemmet Hospital, Oslo, Norway; Ingrid Melle, MD, PhD, NORMENT, KG Jebsen Centre for Psychosis Research, Institute of Clinical Medicine, University of Oslo, and Division of Mental Health and Addiction, Oslo University Hospital; Timothy Hughes, PhD, Department of Medical Genetics, Oslo University Hospital; Ole Andreas Andreassen, MD, PhD, NORMENT, KG Jebsen Centre for Psychosis Research, Institute of Clinical Medicine, University of Oslo, and Division of Mental Health and Addiction, Oslo University Hospital; Srdjan Djurovic, PhD, NORMENT, KG Jebsen Centre for Psychosis Research, Institute of Clinical Medicine, University of Oslo, and Department of Medical Genetics, Oslo University Hospital, Oslo, Norway

Correspondence: Martin Tesli, NORMENT, KG Jebsen Centre for Psychosis Research - TOP Study, Building 49, Oslo University Hospital, Ullevål, Kirkeveien 166, PO Box 4956 Nydalen, 0424 Oslo, Norway. Email: m.s.tesli@medisin.uio.no First received 19 Aug 2013, final revision 23 Jan 2014, accepted 7 Mar 2014

\section{References}

1 Psychiatric GWAS Consortium Bipolar Disorder Working Group. Large-scale genome-wide association analysis of bipolar disorder identifies a new susceptibility locus near ODZ4. Nat Genet 2011; 43: 977-83.

2 Ripke S, Sanders AR, Kendler KS, Levinson DF, Sklar P, Holmans PA, et al. Genome-wide association study identifies five new schizophrenia loci. Nat Genet 2011; 43: 969-76.

3 Roussos P, Katsel P, Davis KL, Bitsios P, Giakoumaki SG, Jogia J, et al. Molecular and genetic evidence for abnormalities in the nodes of Ranvier in schizophrenia. Arch Gen Psychiatry 2012; 69: 7-15.

4 Roussos P, Giakoumaki SG, Georgakopoulos A, Robakis NK, Bitsios P. The CACNA1C and ANK3 risk alleles impact on affective personality traits and startle reactivity but not on cognition or gating in healthy males. Bipolar Disord 2011; 13: 250-9.

5 Linke J, Witt SH, King AV, Nieratschker V, Poupon C, Gass A, et al. Genome-wide supported risk variant for bipolar disorder alters anatomical connectivity in the human brain. Neuroimage 2012; 59: 3288-96.

6 Rueckert EH, Barker D, Ruderfer D, Bergen SE, O'Dushlaine C, Luce CJ, et al. Cis-acting regulation of brain-specific ANK3 gene expression by a genetic variant associated with bipolar disorder. Mol Psychiatry 2013; 18: 922-9.

7 Kato T, Hayashi-Takagi A, Toyota T, Yoshikawa T, Iwamoto K. Gene expression analysis in lymphoblastoid cells as a potential biomarker of bipolar disorder. J Hum Genet 2011; 56: 779-83.

8 Spitzer RL, Williams JB, Gibbon M, First MB. The Structured Clinical Interview for DSM-III-R (SCID). I: History, rationale, and description. Arch Gen Psychiatry 1992; 49: 624-9.

9 Sullivan PF, Fan C, Perou CM. Evaluating the comparability of gene expression in blood and brain. Am J Med Genet B Neuropsychiatr Genet 2006; 141B: 261-8. 